\title{
Hyper Wiener Index of Concatenated Pentagons in Two Rows
}

\author{
M. Srujana ${ }^{1}$, N. Prabhakara Rao ${ }^{2}$ \\ ${ }^{I}$ Asst. Professor/Department of Mathematics/Bapatla Engineering College, Bapatla, India \\ ${ }^{2}$ Professor/Department of Mathematics/ Bapatla Engineering College, Bapatla, India.
}

*Corresponding Author: M. Srujana, Asst. Professor/Department of Mathematics/Bapatla Engineering College, Bapatla, India

Abstract: The major task of QSAR research is to transfer the chemical formula of a molecular graph into numerical format. Topological index is an effective tool to do this. Pentachains in two rows include chemical graph of Dodecahydropentaleno [1,6-cd]pentalene. In this paper, the explicit formulae for hyper wiener index of pentachains in two rows of different lengths are established.

Keywords: Topological index, Hyper wiener.

\section{INTRODUCTION}

A pentachain is a graph formed of concatenated 5-cycles. Ivan Gutman and others [1] in 2005 presented the study of concatenated 5-cycles in one row and obtained explicit formulas for Schultz and modified Szeged indices of pentachains in one row and two rows.

The Hyper wiener index of a graph $\mathrm{G}=(\mathrm{V}, \mathrm{E})$ is defined as $W W(G)=\frac{1}{2} \sum_{i<j}\left(d_{i j}+d_{i j}^{2}\right)$.

\section{NotATiON}

5-cycles can be concatenated in two rows as shown in figures 1,2 (we call these cases as Straight chaining cases) or as in figure 3 (we call this case as Alternate chaining case).

In straight chaining (first case) the graph consisting of 5-cycles in two rows with ' $b$ ' cycles in row 1 and ' $\mathrm{a}$ ' cycles in row 2 denoted by $\mathrm{G}\left(\mathrm{a}, \mathrm{b}, \mathrm{S}_{1}\right)$ as shown below.

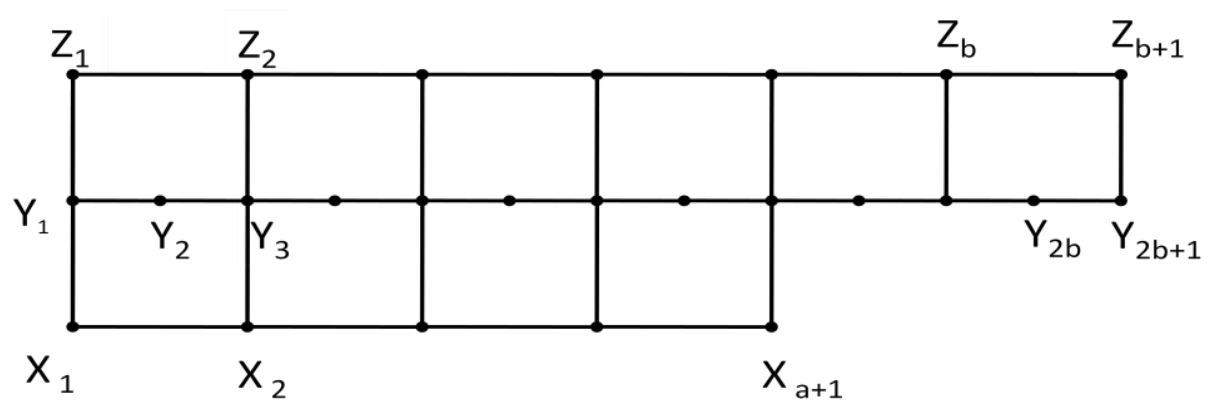

Figure 1. Graph of $\mathrm{G}\left(\mathrm{a}, \mathrm{b}, \mathrm{S}_{1}\right)$

In straight chaining (second case) the graph consisting of 5-cycles in two rows with ' $b$ ' cycles in row 1 and ' $a$ ' cycles in row 2 denoted by $\mathrm{G}\left(\mathrm{a}, \mathrm{b}, \mathrm{S}_{2}\right)$ is as shown below. 


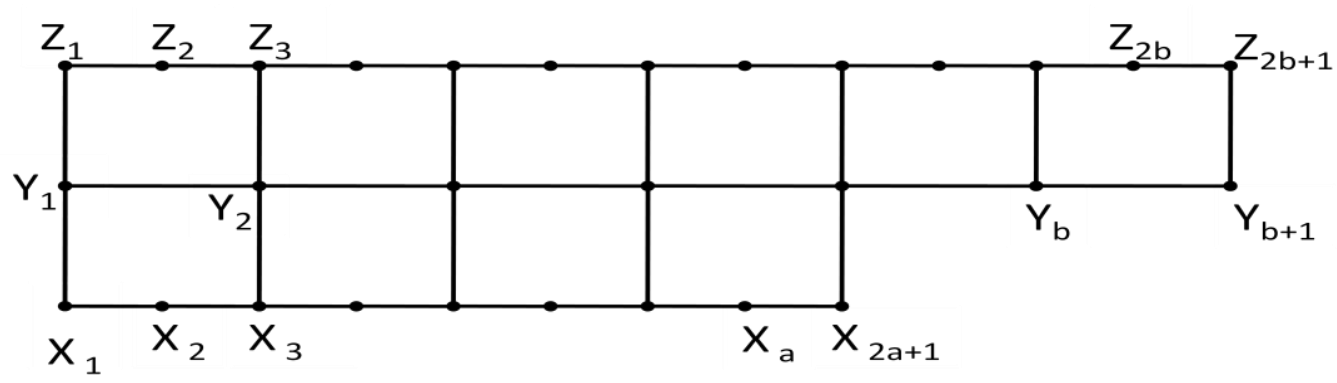

Figure 2. Graph of $\mathrm{G}\left(\mathrm{a}, \mathrm{b}, \mathrm{S}_{2}\right)$

In alternate chaining we denote the graph consisting of 5-cycles in two rows with ' $b$ ' cycles in row 1 and ' $a$ ' cycles in row 2 , as shown below by G(a,b,A).

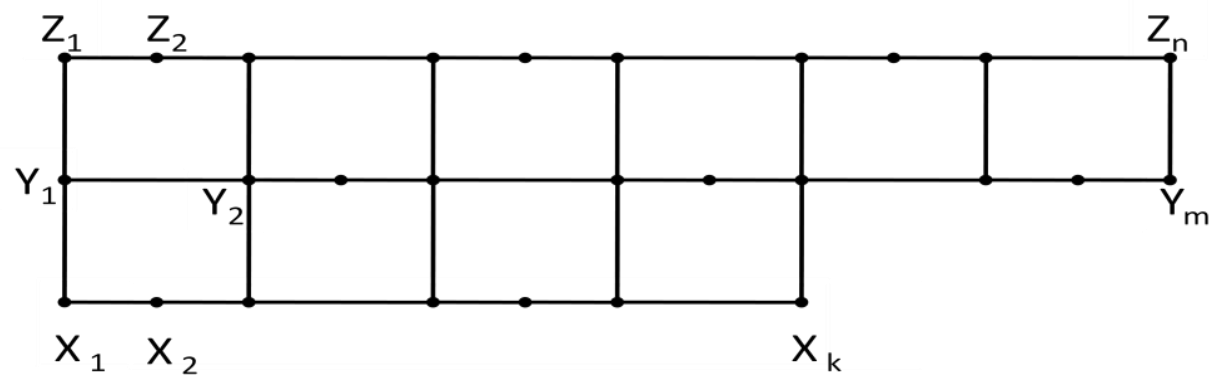

Figure 3. Graph of $\mathrm{G}(\mathrm{a}, \mathrm{b}, \mathrm{A})$

Here, $k=\frac{3 a+2}{2}, m=n=\frac{3 a+2}{2}$, if a is even and $\mathrm{b}$ is even;

$k=\frac{3 a+2}{2}, m=\frac{3 a+1}{2}, n=\frac{3 a+3}{2}$, If $\mathrm{a}$ is even and $\mathrm{b}$ is odd;

$k=\frac{3 a+3}{2}, m=n=\frac{3 a+2}{2}$, If $\mathrm{a}$ is odd and $\mathrm{b}$ is even;

$k=\frac{3 a+3}{2}, m=\frac{3 a+1}{2}, n=\frac{3 a+2}{2}$, if $\mathrm{a}$ is odd and $\mathrm{b}$ is odd.

\section{ALGORITHM}

First we explain the algorithm we adopted for the computation of Hyper wiener index of $G\left(a, b, S_{1}\right)$ with $b>a$.

1. Form the set of vertices $A=\left\{x_{1}, x_{2}, \ldots, x_{a+1}\right\}, B=\left\{y_{1}, y_{2}, \ldots, y_{2 a+1}\right\}, C=\left\{z_{1}, z_{2}, \ldots, z_{a+1}\right\}$ and take $W W=0$.

2. Compute the Hyper wiener index ( say $_{1}$ ) for the sub graph generated by $B \cup C$ using the formula $W W(G(a, s))$ and $W W=W W+n_{1}$.

3. Compute $\sum_{u, v \in A} d(u, v)$, the sum of the shortest distances between vertices of $\mathrm{A}$ (say $\mathrm{n}_{2}$ ), $W W=W W+\frac{n_{2}}{2}$. 
4. Compute $\sum_{u \in A, v \in B} d(u, v)$, the sum of the shortest distances between vertices of $\mathrm{A}$ and $\mathrm{B}$ $\left(\right.$ say $\left.\mathrm{n}_{3}\right), W W=W W+\frac{n_{3}}{2}$.

5. Compute $\sum_{u \in A, v \in C} d(u, v)$, the sum of the shortest distances between vertices of $\mathrm{A}$ and $\mathrm{C}$ $\left(\right.$ say $\left.\mathrm{n}_{4}\right), W W=W W+\frac{n_{4}}{2}$.

6. Compute $\sum_{u, v \in A} d^{2}(u, v)$, the squares of the sum of the shortest distances between vertices of $\mathrm{A}$ $\left(\right.$ say $\left.\mathrm{n}_{5}\right), W W=W W+\frac{n_{5}}{2}$.

7. Compute $\sum_{u \in A, v \in B} d^{2}(u, v)$, the squares of the sum of the shortest distances between vertices of $\mathrm{A}$ and $\mathrm{B}\left(\right.$ say $\left._{6}\right), W W=W W+\frac{n_{6}}{2}$.

8. Compute $\sum_{u \in A, v \in C} d^{2}(u, v)$, the squares of the sum of the shortest distances between vertices of $\mathrm{A}$ and $\mathrm{C}\left(\right.$ say $\left._{7}\right), W W=W W+\frac{n_{7}}{2}$.

9. $W W$ is the Hyper wiener index of the graph $G\left(a, b, S_{1}\right)$.

By modifying the above algorithms suitably, Hyper wiener index for all other cases can be computed.

\section{Main Results}

\section{Theorem 4.1.}

$$
\begin{aligned}
W W\left(G\left(a, b, S_{1}\right)\right) & =53 \text { if } a=b=1 . \\
& =165 \text { if } a=1, b=2 . \\
& =\frac{1}{6}\left(4 a^{4}+44 a^{3}+182 a^{2}-62 a+180\right) \text { if } a=b \geq 2 . \\
& =\frac{1}{6}\left(4 a^{4}+56 a^{3}+305 a^{2}+361 a+264\right) \text { if } b=a+1, a \geq 2 . \\
& =\frac{1}{8}\left(3 b^{4}+42 b^{3}+221 b^{2}+54 b-144\right) \text { if } a=1, b>2 .
\end{aligned}
$$

Proof: For $a=b=1$ and $b=a+1=2$ the results are trivial. From the following lemmas (4.2 to 4.4) the proof of the theorem is clear.

Lemma 4.2. $W W\left(G\left(a, a, S_{1}\right)\right)=\frac{1}{6}\left(4 a^{4}+44 a^{3}+182 a^{2}-62 a+180\right)$ if $a \geq 2$.

Proof: Let the vertex set of $G\left(a, a, S_{1}\right)$ be $A \cup B \cup C$, where

$$
A=\left\{x_{1}, x_{2}, \ldots, x_{a+1}\right\}, B=\left\{y_{1}, y_{2}, \ldots, y_{2 a+1}\right\}, C=\left\{z_{1}, z_{2}, \ldots, z_{a+1}\right\} \text {. }
$$


Now

$$
\begin{aligned}
W W\left(G\left(a, a, S_{1}\right)\right)= & W W(G(a, S))+\frac{1}{2}\left\{\sum_{u, v \in A} d(u, v)+\sum_{u \in A, v \in B} d(u, v)+\sum_{u \in A, v \in C} d(u, v)\right. \\
& \left.+\sum_{u, v \in A} d^{2}(u, v)+\sum_{u \in A, v \in B} d^{2}(u, v)+\sum_{u \in A, v \in C} d^{2}(u, v)\right\} . \\
= & \frac{1}{8}\left(3 a^{4}+34 a^{3}+145 a^{2}-190 a+208\right)+\frac{1}{2}\left(\left\{\sum_{i=1}^{a}(a-(i-1))(i)\right\}+\{(a+1)(1)+\right. \\
& \left.\sum_{i=2}^{a}(a-(i-2))(4 i)+4(a+1)\right\}+\left\{(a+1)(2)+\sum_{i=3}^{a+1}(2 a-(2 i-6))(i)+2(a+2)\right\} \\
& +\left\{\sum_{i=1}^{a}(a-(i-1))(i)^{2}\right\}+\left\{(a+1)(1)^{2}+4 \sum_{i=2}^{a}(a-(i-2))(i)^{2}+4(a+1)^{2}\right\} \\
+ & \left.\left\{(a+1)(2)^{2}+\sum_{i=3}^{a+1}(2 a-(2 i-6))(i)^{2}+2(a+2)^{2}\right\}\right) . \\
= & \frac{1}{8}\left(3 a^{4}+34 a^{3}+145 a^{2}-190 a+208\right)+\frac{1}{2}\left\{\frac{1}{6}\left(a^{3}+3 a^{2}+2 a\right)+\right. \\
& \frac{1}{3}\left(2 a^{3}+12 a^{2}+13 a+3\right)+\frac{1}{3}\left(a^{3}+9 a^{2}+14 a+6\right)+\frac{1}{12}\left(a^{4}+4 a^{3}+5 a^{2}+2 a\right)+ \\
& \left.\frac{1}{3}\left(a^{4}+8 a^{3}+23 a^{2}+19 a+3\right)+\frac{1}{6}\left(a^{4}+12 a^{3}+53 a^{2}+66 a+24\right)\right\} . \\
= & \frac{1}{6}\left(4 a^{4}+44 a^{3}+182 a^{2}-62 a+180\right) .
\end{aligned}
$$

Lemma 4.3. $W W\left(G\left(a, a+1, S_{1}\right)\right)=\frac{1}{6}\left(4 a^{4}+56 a^{3}+305 a^{2}+361 a+264\right)$ if $b=a+1, a \geq 2$.

Proof: Let the vertex set of $G\left(a, a+1, S_{1}\right)$ be $A \cup B \cup C$, where $A=\left\{x_{1}, x_{2}, \ldots, x_{a+1}\right\}, B=\left\{y_{1}, y_{2}, \ldots, y_{2 a+1}\right\}, C=\left\{z_{1}, z_{2}, \ldots, z_{a+1}\right\}$.

Now

$$
\begin{aligned}
W W\left(G\left(a, a+1, S_{1}\right)\right)= & W W(G(b, S))+\frac{1}{2}\left\{\sum_{u, v \in A} d(u, v)+\sum_{u \in A, v \in B} d(u, v)+\sum_{u \in A, v \in C} d(u, v)\right. \\
& \left.+\sum_{u, v \in A} d^{2}(u, v)+\sum_{u \in A, v \in B} d^{2}(u, v)+\sum_{u \in A, v \in C} d^{2}(u, v)\right\} . \\
= & \frac{1}{8}\left(3 a^{4}+34 a^{3}+145 a^{2}-190 a+208\right)+\frac{1}{2}\left(\left\{\sum_{i=1}^{a}(a-(i-1))(i)\right\}+\{(a+1)(1)\right. \\
& \left.+(4 a+1)(2)+\sum_{i=3}^{a+2}(4 a-(4 i-10))(i)+(1)(a+3)\right\}+\{(a+1)(2)+ \\
& \left.+\sum_{i=3}^{a+1}(2 a-(2 i-7))(i)\right\}+\left\{\sum_{i=1}^{a}(a-(i-1))(i)^{2}\right\}+\left\{(a+1)(1)^{2}+(4 a+1)\left(2^{2}\right)\right. \\
& \left.\left.+\sum_{i=3}^{a+2}(4 a-(4 i-10))(i)^{2}+1(a+3)^{2}\right\}+\left\{(a+1)(2)^{2}+\sum_{i=3}^{a+1}(2 a-(2 i-7))(i)^{2}\right\}\right) .
\end{aligned}
$$




$$
\begin{aligned}
& =\frac{1}{8}\left(3 a^{4}+34 a^{3}+145 a^{2}-190 a+208\right)+\frac{1}{2}\left\{\frac{1}{6}\left(a^{3}+3 a^{2}+2 a\right)\right. \\
& +\frac{1}{3}\left(2 a^{3}+15 a^{2}+31 a+18\right)+\frac{1}{3}\left(8 a^{3}+72 a^{2}+124 a+81\right)+\frac{1}{12}\left(a^{4}+4 a^{3}+5 a^{2}+2 a\right) \\
& \left.+\frac{1}{3}\left(a^{4}+10 a^{3}+41 a^{2}+74 a+42\right)+\frac{1}{6}\left(a^{4}+14 a^{3}+74 a^{2}+139 a+78\right)\right\} . \\
& =\frac{1}{6}\left(4 a^{4}+56 a^{3}+305 a^{2}+361 a+264\right) .
\end{aligned}
$$

Lemma 4.4. $W W\left(G\left(1, b, S_{1}\right)\right)=\frac{1}{8}\left(3 b^{4}+42 b^{3}+221 b^{2}+54 b-144\right)$ if $a=1, b>2$.

Proof: Let the vertex set of $G\left(1, b, S_{1}\right)$ be $A \cup B \cup C$, where

$$
A=\left\{x_{1}, x_{2}\right\}, B=\left\{y_{1}, y_{2}, \ldots, y_{2 b+1}\right\}, C=\left\{z_{1}, z_{2}, \ldots, z_{b+1}\right\} \text {. }
$$

Now

$$
\begin{aligned}
W W\left(G\left(1, b+1, S_{1}\right)\right)= & W W(G(b, S))+\frac{1}{2}\left\{\sum_{u, v \in A} d(u, v)+\sum_{u \in A, v \in B} d(u, v)+\sum_{u \in A, v \in C} d(u, v)\right. \\
& \left.+\sum_{u, v \in A} d^{2}(u, v)+\sum_{u \in A, v \in B} d^{2}(u, v)+\sum_{u \in A, v \in C} d^{2}(u, v)\right\} . \\
= & \frac{1}{8}\left(3 a^{4}+34 a^{3}+145 a^{2}-190 a+208\right)+\frac{1}{2}\left(1+\left\{2(1)+5(2)+2 \sum_{i=3}^{5} i+3(6)\right.\right. \\
& \left.+4 \sum_{i=7}^{b+2} i+2(b+3)\right\}+\left\{2 \sum_{i=1}^{b+1} i+(b+3)\right\}+\left\{2(1)^{2}+5(2)^{2}+2 \sum_{i=3}^{5} i^{2}+3(6)^{2}\right. \\
& \left.+4 \sum_{i=7}^{b+2} i^{2}+2(b+3)^{2}\right\}+\left\{2(2)^{2}+3(3)^{2}+2 \sum_{i=4}^{b+1} i^{2}+(b+2)^{2}\right\} . \\
= & \frac{1}{8}\left(3 a^{4}+34 a^{3}+145 a^{2}-190 a+208\right)+\frac{1}{2}\left\{\left(1+\left(2 b^{2}+12 b-12\right)+\left(b^{2}+4 b+5\right)\right.\right. \\
& +1+\frac{1}{3}\left(4 b^{3}+36 b^{2}+110 b-288\right)+\frac{1}{3}\left(2 b^{3}+12 b^{2}+25 b+39\right) . \\
= & \frac{1}{8}\left(3 b^{4}+42 b^{3}+221 b^{2}+54 b-144\right) .
\end{aligned}
$$

\section{Theorem 4.5.}

$W W\left(G\left(a, b, S_{2}\right)\right)=91$ if $a=b=1$.

$$
\begin{aligned}
& =\frac{1}{24}\left(25 a^{4}+310 a^{3}+1427 a^{2}-658 a+1320\right) \text { if } a=b \geq 2 . \\
& =\frac{1}{8}\left(3 b^{4}+46 b^{3}+229 b^{2}+10 b+480\right) \text { if } a=1, b>1 . \\
& =\frac{1}{8}\left(3 b^{4}+56 b^{3}+257 b^{2}+46 b+1264\right) \text { if } a=2, b>1 .
\end{aligned}
$$

Proof: For $a=b=1$ the result is trivial. From the following lemmas ( 4.6 to 4.8 ) the proof of the theorem is clear. 
Lemma 4.6. $W W\left(G\left(a, a, S_{2}\right)\right)=\frac{1}{24}\left(25 a^{4}+310 a^{3}+1427 a^{2}-658 a+1320\right)$ if $a \geq 2$.

Proof: Let the vertex set of $G\left(a, a, S_{2}\right)$ be $A \cup B \cup C$, where

$$
A=\left\{x_{1}, x_{2}, \ldots, x_{2 a+1}\right\}, B=\left\{y_{1}, y_{2}, \ldots, y_{a+1}\right\}, C=\left\{z_{1}, z_{2}, \ldots, z_{2 a+1}\right\}
$$

Now

$$
\begin{aligned}
W W\left(G\left(a, a, S_{2}\right)\right)= & W W(G(a, S))+\frac{1}{2}\left\{\sum_{u, v \in A} d(u, v)+\sum_{u \in A, v \in B} d(u, v)+\sum_{u \in A, v \in C} d(u, v)\right. \\
& \left.+\sum_{u, v \in A} d^{2}(u, v)+\sum_{u \in A, v \in B} d^{2}(u, v)+\sum_{u \in A, v \in C} d^{2}(u, v)\right\} . \\
= & \frac{1}{8}\left(3 a^{4}+34 a^{3}+145 a^{2}-190 a+208\right)+\frac{1}{2}\left(\left\{\sum_{i=1}^{4}(2 a-(i-1))(i)+(3 a-6)(5)\right.\right. \\
& \left.+\sum_{i=6}^{a+1}(4 a-(4 i-12))(i)+4(a+2)\right\}+\left\{(a+1)(1)+4 \sum_{i=2}^{a+1}(a-(i-2))(i)\right\} \\
& +\left\{(a+1)(2)+6 a(3)+(9 a-8)(4)+8 \sum_{i=5}^{a+2}(a-(i-3))(i)\right\}+\left\{\sum_{i=1}^{4}(2 a-(i-1))(i)^{2}\right. \\
+ & \left.(3 a-6)(5)^{2}+\sum_{i=6}^{a+1}(4 a-(4 i-12))(i)^{2}+4(a+2)^{2}\right\}+\left\{(a+1)(1)^{2}+\right. \\
& \left.\left.\sum_{i=2}^{a+2} 4(a-(i-2))(i)^{2}\right\}+\left\{(a+1)(2)^{2}+6 a(3)^{2}+(9 a-8)(4)^{2}+8 \sum_{i=5}^{a+2}(a-(i-3))(i)^{2}\right\}\right) . \\
= & \frac{1}{8}\left(3 a^{4}+34 a^{3}+145 a^{2}-190 a+208\right)+\frac{1}{2}\left\{\frac{1}{6}\left(2 a^{3}+18 a^{2}-23 a+18\right)\right. \\
& +\frac{1}{3}\left(2 a^{3}+12 a^{2}+13 a+3\right)+\frac{1}{3}\left(4 a^{3}+36 a^{2}+32 a+6\right)+\frac{1}{3}\left(a^{4}+12 a^{3}+53 a^{2}-153 a+132\right) \\
& \left.+\frac{1}{3}\left(a^{4}+8 a^{3}+23 a^{2}+19 a+3\right)+\frac{1}{3}\left(2 a^{4}+24 a^{3}+106 a^{2}+90 a+12\right)\right\} . \\
= & \frac{1}{24}\left(25 a^{4}+310 a^{3}+1427 a^{2}-658 a+1320\right) .
\end{aligned}
$$

Lemma 4.7. $W W\left(G\left(1, b, S_{2}\right)\right)=\frac{1}{8}\left(3 b^{4}+46 b^{3}+229 b^{2}+10 b+480\right)$ if $b>1$.

Proof: Let the vertex set of $G\left(1, b, S_{2}\right)$ be $A \bigcup B \bigcup C$, where

$$
A=\left\{x_{1}, x_{2}, x_{3}\right\}, B=\left\{y_{1}, y_{2}, \ldots, y_{b+1}\right\}, C=\left\{z_{1}, z_{2}, \ldots, z_{2 b+1}\right\}
$$

Now

$$
\begin{aligned}
W W\left(G\left(1, b, S_{2}\right)\right)= & W W(G(b, S))+\frac{1}{2}\left\{\sum_{u, v \in A} d(u, v)+\sum_{u \in A, v \in B} d(u, v)+\sum_{u \in A, v \in C} d(u, v)\right. \\
& \left.+\sum_{u, v \in A} d^{2}(u, v)+\sum_{u \in A, v \in B} d^{2}(u, v)+\sum_{u \in A, v \in C} d^{2}(u, v)\right\} . \\
= & \frac{1}{8}\left(3 a^{4}+34 a^{3}+145 a^{2}-190 a+208\right)+\frac{1}{2}\left(4+\left\{2(1)+5(2)+3 \sum_{i=3}^{b}(i)+2(b+1)\right\}\right.
\end{aligned}
$$




$$
\begin{aligned}
& +\left\{2(2)+8(3)+7(4)+6 \sum_{i=5}^{b+1}(i)+4(b+2)\right\}+\left\{6+2(1)^{2}+5(2)^{2}+\sum_{i=3}^{b} i^{2}+2(b+1)^{2}\right\} \\
& \left.+\left\{2(2)^{2}+8(3)^{2}+7(4)^{2}+6 \sum_{i=5}^{b+1} i^{2}+4(b+2)^{2}\right\}\right) . \\
& =\frac{1}{8}\left(3 a^{4}+34 a^{3}+145 a^{2}-190 a+208\right)+\frac{1}{2}\left\{4+\frac{1}{2}\left(3 b^{2}+7 b+10\right)+\left(3 b^{2}+13 b+10\right)\right. \\
& \left.+(6)+\frac{1}{2}\left(2 b^{3}+7 b^{2}+9 b+18\right)+\left(2 b^{3}+13 b^{2}+29 b+34\right)\right\} . \\
& =\frac{1}{8}\left(3 b^{4}+46 b^{3}+229 b^{3}+10 b+480\right) .
\end{aligned}
$$

Lemma 4.8. $W W\left(G\left(2, b, S_{2}\right)\right)=\frac{1}{8}\left(3 b^{4}+56 b^{3}+257 b^{2}+46 b+1264\right)$ if $b>1$.

Proof: Let the vertex set of ` be $A \cup B \cup C$, where

$$
A=\left\{x_{1}, x_{2}, \ldots, x_{5}\right\}, B=\left\{y_{1}, y_{2}, \ldots, y_{b+1}\right\}, C=\left\{z_{1}, z_{2}, \ldots, z_{2 b+1}\right\} \text {. }
$$

Now

$$
\begin{aligned}
& W W\left(G\left(2, b, S_{2}\right)\right)=W W(G(b, S))+\frac{1}{2}\left\{\sum_{u, v \in A} d(u, v)+\sum_{u \in A, v \in B} d(u, v)+\sum_{u \in A, v \in C} d(u, v)\right. \\
&\left.+\sum_{u, v \in A} d^{2}(u, v)+\sum_{u \in A, v \in B} d^{2}(u, v)+\sum_{u \in A, v \in C} d^{2}(u, v)\right\} . \\
&= \frac{1}{8}\left(3 a^{4}+34 a^{3}+145 a^{2}-190 a+208\right)+\frac{1}{2}(\{20\}+\{(a+1)(1)+(4 a+1)(2) \\
&\left.+(4 a-1)(3)+5 \sum_{i=4}^{b-1}(i)+4 b+2(b+1)\right\}+\{(a+1)(2)+7 a(3)+8 a(4) \\
&\left.+10 \sum_{i=5}^{b}(i)+8(b+1)+4(b+2)\right\}+\left\{\sum_{i=1}^{4}(2 b-(i-1))(i)^{2}+(3 b-6)(5)^{2}\right. \\
&\left.+\sum_{i=6}^{b+1}(4 b-(4 i-12))(i)^{2}+4(b+2)^{2}\right\}+\left\{(a+1)(1)^{2}+(4 a+1)(2)^{2}\right. \\
&\left.+(4 a-1)(3)^{2}+5 \sum_{i=4}^{b-1}(i)^{2}+4 b^{2}+2(b+1)^{2}\right\}+\left\{(a+1)(2)^{2}+7 a(3)^{2}\right. \\
&\left.\left.+8 a(4)^{2}+10 \sum_{i=5}^{b}(i)^{2}+8(b+1)^{2}+4(b+2)^{2}\right\}\right) . \\
&= \frac{1}{8}\left(3 a^{4}+34 a^{3}+145 a^{2}-190 a+208\right)+\frac{1}{2}\left\{20+\frac{1}{2}\left(5 b^{2}+7 b+28\right)\right. \\
&+\left(5 b^{2}+17 b+28\right)+(50)+\frac{1}{6}\left(10 b^{3}+21 b^{2}+29 b+204\right) \\
&\left.+\frac{1}{3}\left(10 b^{3}+51 b^{2}+101 b+354\right)\right\} . \\
&= \frac{1}{8}\left(3 b^{4}+54 b^{3}+257 b^{2}+46 b+1264\right) .
\end{aligned}
$$




\section{Theorem 4.9.}

$$
\begin{aligned}
W W(G(a, b, A)) & =\frac{1}{128}\left(243 a^{4}+1368 a^{3}+2826 a^{2}+7784 a-573\right), \text { where } a=b \text { is odd } . \\
& =\frac{1}{128}\left(243 a^{4}+1260 a^{3}+2340 a^{2}+7088 a-2432\right), \text { where } a=b \text { is even } .
\end{aligned}
$$

Proof: The proof is clear from the following lemmas (4.10 to 4.11).

Lemma 4.10. $W W(G(a, a, A))=\frac{1}{128}\left(243 a^{4}+1368 a^{3}+2826 a^{2}+7784 a-573\right)$, where a is odd.

Proof: Let the vertex set of $G(a, a, A)$ be The $A \cup B \cup C$, where

$$
A=\left\{x_{1}, x_{2}, \ldots, \frac{x_{\frac{3 a+3}{2}}}{2}\right\}, B=\left\{y_{1}, y_{2}, \ldots, y_{\frac{3 a+1}{2}}\right\}, C=\left\{z_{1}, z_{2}, \ldots, z_{\frac{3 a+3}{2}}\right\} \text {. }
$$

Now

$$
\begin{aligned}
W W(G(a, a, A))= & W W(G(a, A))+\frac{1}{2}\left\{\sum_{u, v \in A} d(u, v)+\sum_{u \in A, v \in B} d(u, v)+\sum_{u \in A, v \in C} d(u, v)\right. \\
& \left.+\sum_{u, v \in A} d^{2}(u, v)+\sum_{u \in A, v \in B} d^{2}(u, v)+\sum_{u \in A, v \in C} d^{2}(u, v)\right\} . \\
= & \frac{1}{32}\left(27 a^{4}+126 a^{3}+204 a^{2}+354 a-71\right)+\frac{1}{2}\left(\left\{\sum_{i=1}^{\frac{3 a+1}{2}}\left(\frac{3 a+1}{2}-(i-1)\right)(i)\right\}+\{(a+1)(1)\right. \\
& \left.+4 a(2)+(4 a-4)(3)+\sum_{i=4}^{\frac{3 a+1}{2}}((3 a-5)-(2 i-8))(i)\right\}+\{(a+1)(2)+(4 a+2)(3) \\
& \left.+\frac{1}{2}(9 a-7)(4)+(4 a-6)(5)+\sum_{i=6}^{\frac{3 a+3}{2}}((3 a-7)-(2 i-12))(i)\right\}+\left\{\sum_{i=1}^{2}\left(\frac{3 a+1}{2}-(i-1)(i)^{2}\right)\right\} \\
+ & \left\{(a+1)(1)^{2}+4 a(2)^{2}+(4 a-4)(3)^{2}+\sum_{i=4}^{\frac{3 a+1}{2}}\left((3 a-5)-(2 i-8)(i)^{2}\right)\right\}+\left\{(a+1)(2)^{2}\right. \\
& \left.\left.+(4 a+2)(3)^{2}+\frac{1}{2}(9 a-7)(4)^{2}+(4 a-6)(5)^{2}+\sum_{i=6}^{\frac{3 a+3}{2}}((3 a-7)-(2 i-12))(i)^{2}\right\}\right) . \\
= & \frac{1}{32}\left(27 a^{4}+126 a^{3}+204 a^{2}+354 a-71\right)+\frac{1}{2}\left\{\frac{1}{16}\left(9 a^{3}+27 a^{2}+23 a+5\right)\right. \\
& +\frac{1}{8}\left(9 a^{3}+27 a^{2}+47 a-3\right)+\frac{1}{8}\left(9 a^{3}+45 a^{2}+127 a+27\right) \\
& \left.+\frac{1}{64}\left(27 a^{4}+108 a^{3}+150 a^{2}+84 a+15\right)+\frac{1}{32}\left(27 a^{4}+108 a^{3}+438 a^{2}+1964 a-113\right)\right\} . \\
= & \frac{1}{128}\left(243 a^{4}+1368 a^{3}+2826 a^{2}+7784 a-573\right) .
\end{aligned}
$$

Lemma 4.11. $W W(G(a, a, A))=\frac{1}{128}\left(243 a^{4}+1260 a^{3}+2340 a^{2}+7088 a-2432\right)$, where a is even.

Proof: Let the vertex set of $G(a, a, A)$ be The $A \cup B \cup C$, where

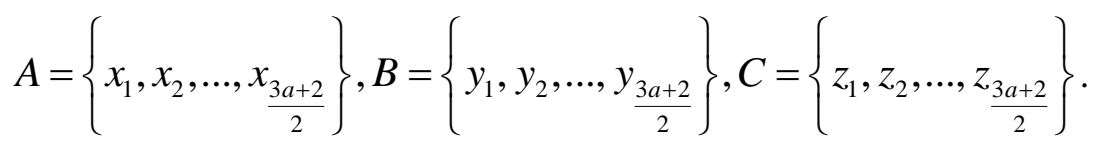




$$
\begin{aligned}
\text { Now } & \\
W W(G(a, a, A))= & W W(G(a, A))+\frac{1}{2}\left\{\sum_{u, v \in A} d(u, v)+\sum_{u \in A, v \in B} d(u, v)+\sum_{u \in A, v \in C} d(u, v)\right. \\
& \left.+\sum_{u, v \in A} d^{2}(u, v)+\sum_{u \in A, v \in B} d^{2}(u, v)+\sum_{u \in A, v \in C} d^{2}(u, v)\right\} . \\
= & \frac{1}{32}\left(27 a^{4}+126 a^{3}+204 a^{2}+360 a-64\right)+\frac{1}{2}\left(\left\{\sum_{i=1}^{\frac{3 a}{2}}\left(\frac{3 a}{2}-(i-1)\right)(i)\right\}+\{(a+1)(1)\right. \\
& \left.+4 a(2)+(4 a-4)(3)+\sum_{i=4}^{\frac{3 a-4}{2}}((3 a)-(2 i-3))(i)+5\left(\frac{3 a-2}{2}\right)+3\left(\frac{3 a}{2}\right)+\left(\frac{3 a+2}{2}\right)\right\} \\
& +\left\{(a+1)(2)+(4 a)(3)+\left(\frac{9 a-8}{2}\right)(4)+(4 a-8)(5)+\sum_{i=6}^{\frac{3 a+2}{2}}((3 a-8)-((2 i-12))(i))\right\} \\
& +\left\{\sum_{i=1}^{\frac{3 a}{2}}\left(\frac{3 a}{2}-(i-1)\right)(i)^{2}\right\}+\left\{(a+1)(1)^{2}+4 a(2)^{2}+(4 a-4)(3)^{2}+\right. \\
& \left.\frac{3 a-4}{2}\left(3 a-(2 i-3)(i)^{2}\right)+5\left(\frac{3 a-2}{2}\right)^{2}+\left(\frac{3 a}{2}\right)^{2}+\left(\frac{3 a+2}{2}\right)^{2}\right\}+\left\{(a+1)(1)^{2}+4 a(2)^{2}\right. \\
& \sum_{i=4}(3 a-3)^{2} \\
& \left.\left.+(4 a-4)(3)^{2}+\sum_{i=4}^{2}(3 a-(2 i-3))(i)^{2}\right\}+5\left(\frac{3 a-2}{2}\right)^{2}+3\left(\frac{3 a}{2}\right)^{2}+\left(\frac{3 a+2}{2}\right)^{2}\right\} \\
= & \frac{1}{128}\left(243 a^{4}+1260 a^{3}+2340 a^{2}+7088 a-2432\right) . \\
& \left.+\left\{(a+1)(2)^{2}+4 a(3)^{2}+\left(\frac{9 a-8}{2}\right)(4)^{2}+(4 a-8)(5)^{2}+\sum_{i=6}^{2}((3 a-8)-(2 i-12))(i)^{2}\right\}\right) . \\
= & \frac{1}{32}\left(27 a^{4}+126 a^{3}+204 a^{2}+360 a-64\right)+\frac{1}{2}\left\{\frac{1}{16}\left(9 a^{3}+18 a^{2}+8 a\right)+\frac{1}{8}\left(9 a^{3}+27 a^{2}+50 a\right)\right. \\
& +\frac{1}{8}\left(9 a^{3}+36 a^{2}+100 a-16\right)+\frac{1}{64}\left(27 a^{4}+72 a^{3}+60 a^{2}+16 a\right)+ \\
& \\
& \\
&
\end{aligned}
$$

\section{CONCLUSION}

The effective explicit formulas for Hyper Wiener Index of pentachains are determined successfully in two rows of different lengths. It is also established efficiently the transformation of chemical formula into numerical formula for the purpose of an analytical study. Moreover the pentachains in two rows has been established with the chemical graph of Dodecahydropentaleno[1,6-cd]pentalene.

\section{REFERENCES}

[1] Gutman I, Yan W, Yeh, Y.N, Yang.B, Generalized Wiener index of Zigzagging Pentachains, Journal of Mathematical Chemistry, Vol 42,No.2, P 103-117,2007.

[2] Prabhakara Rao N, Lavanya Lakshmi K, Laxmi Prasanna A, On the Wiener index of Penta chains, proceeding of national conference on discrete mathematics and its applications, NCDMA, Thiagarajar college of engineering, Madurai, 2007.

[3] Prabhakara Rao N, Laxmi Prasanna A, On PI index of Penta chains, Acta Ciencia Indica, Vol.XXXVM, No.3, 1117(2009). 


\section{AUTHOR'S BIOGRAPHY}

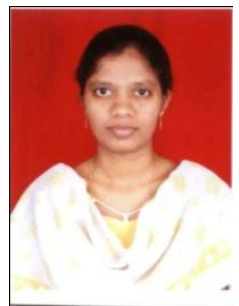

M. Srujana, has been working as Asst.Prof in Dept. of Mathematics from past 10 years at Bapatla Engineering College. She published four journals in the area of Graph Theory. At present she is pursing P.hd at Acharya Nagarjuna University. Her areas of research interests are Graph Theory and Algebra.

N. Prabhakara Rao, worked for 37 years as Professor \& Head of Dept. Mathematics at Bapatla Engineering College. He obtained P.hd from Andhra University. He guided several research scholars in the areas of Mathematics-Lattice Theory, Graph Theory \& Algebra. He is a life member of International Society for Technology in Education. He has 14 International and 10 National publications. The students of him were currently working at several prestigious organizations occupying eminent positions.

Citation: M. Srujana, N. Prabhakara Rao, "Hyper Wiener Index of Concatenated Pentagons in Two Rows ", International Journal of Scientific and Innovative Mathematical Research, vol. 6, no. 3, p. 1-10, 2018., http://dx.doi.org/10.20431/2347-3142.0603001

Copyright: (C) 2018 Authors. This is an open-access article distributed under the terms of the Creative Commons Attribution License, which permits unrestricted use, distribution, and reproduction in any medium, provided the original author and source are credited. 